\title{
Activated STAT3 Could Reduce Survival in Patients with Esophageal Squamous Cell Carcinoma by Up-regulating VEGF and Cyclin D1 Expression
}

\author{
Nan Zhang1, Min Zhang², Zhou Wang 3 , Wei Gao ${ }^{4}$, Zhi-Gang Sun ${ }^{5}$ \\ 1. Department of Oncology, Jinan Central Hospital Affiliated to Shandong University; Jinan Central Hospital Affiliated to Shandong First Medical University; \\ Jinan 250013, P.R. China. \\ 2. Department of Dermatology, Jinan Central Hospital Affiliated to Shandong University; Jinan Central Hospital Affiliated to Shandong First Medical \\ University; Jinan 250013, P.R. China. \\ 3. Department of Thoracic Surgery, Provincial Hospital Affiliated to Shandong University, Jinan 250021, P.R. China. \\ 4. Department of Pathology, Jinan Central Hospital Affiliated to Shandong University; Jinan Central Hospital Affiliated to Shandong First Medical University; \\ Jinan 250013, P.R. China. \\ 5. Department of Thoracic Surgery, Jinan Central Hospital Affiliated to Shandong University; Jinan Central Hospital Affiliated to Shandong First Medical \\ University; Jinan 250013, P.R. China. \\ $\square$ Corresponding author: Zhi-Gang Sun, Department of Thoracic Surgery, Jinan Central Hospital Affiliated to Shandong University; Jinan Central Hospital \\ Affiliated to Shandong First Medical University; Jinan 250013, P.R. China. Phone: +8613370582825. E-mail: szg@zxyy.cn.
}

( ) The author(s). This is an open access article distributed under the terms of the Creative Commons Attribution License (https://creativecommons.org/licenses/by/4.0/). See http:/ /ivyspring.com/terms for full terms and conditions.

Received: 2019.07.28; Accepted: 2019.12.01; Published: 2020.01.20

\begin{abstract}
Signal transduction and activators of transcription factor (STAT) 3 is associated with a poor prognosis in certain types of cancer. The purpose of the present study was to investigate the clinical and prognostic significance of STAT3/p-STAT3 expression in esophageal squamous cell cancer (ESCC) patients. A total of 71 patients were enrolled in the study. STAT3 and p-STAT3 expression were detected by Western Blot and immunohistochemistry assays. Stattic, the STAT3 inhibitor, was used to block the activation of STAT3 in ESCC cell lines Eca-109 and Kyse-30, and the CCK8 assay was performed to detect the effect of Stattic on the viability of ESCC cells. The expression of associated genes was assessed by RT-PCR and Western blot at RNA and protein levels, respectively. STAT3 expression was correlated with infiltration degree (PT) and PTNM. And $\mathrm{p}$-STAT3 expression was correlated with $\mathrm{pT}$, lymphatic metastasis $(\mathrm{pN})$ and $\mathrm{PTNM}$. The expression of VEGF, Bcl-xl and Cyclin DI was up-regulated in ESCC tissues and positively correlated with p-STAT3 level, besides Bcl-xl. In vitro, Stattic inhibited the viability of Eca-109 and Kyse-30 cells in a dose- and time- dependent manner, and significantly inhibited the expression of VEGF, Bcl-xl and CyclinDI at mRNA and protein level. The 5 -year survival rate of the 71 patients was significantly associated with pT, pN, pTNM stage, p-STAT3 level, VEGF expression and Cyclin DI expression. $\mathrm{pN}$ and $\mathrm{p}$-STAT3 expression were independent relevant factors. Our results showed that p-STAT3 might serve as an essential biomarker for tumor invasion and metastasis in ESCC.
\end{abstract}

Key words: STAT3, p-STAT3, Esophageal squamous cell cancer, Western Blot, Immunohistochemistry.

\section{Introduction}

Esophageal cancer is one of the ten malignant tumors in China, of which esophageal squamous cell carcinoma (ESCC) is the major histological type. Esophagostomy is the major treatment method at present. However, the prognosis is not satisfactory, and the 5-year survival rate of the ESCC patients is less than $30 \%$ [1]. The tumor-node-metastasis (TNM) staging system, according to histopathologic findings, lacks sufficient predictive value as significant differences in survival are often observed for the same TNM stage. Therefore, a combination of a number of biomarkers to more accurately distinguish patients with ESCC with poor survival would be valuable [2, 3]. 
Signal transduction and activators of transcription factor (STAT) 3, a member of the STAT family, is frequently regarded as an oncogene [4-6]. STAT3 can be activated via phosphorylation events within the Janus kinase-STAT, producing tyrosine or serine phosphorylated STAT3 (p-STAT3) [7, 8]. p-STAT3 is able to increase the expression levels of certain target genes, including vascular endothelial growth factor (VEGF), Cyclin D1 and Bcl-xL, etc [9-12]. STAT3 constitutively activation is frequently observed in cancers, including lung cancer, liver cancer, gastric cancer and renal cell carcinoma [13]. Increasing number of studies show that STAT3 may serve as one of the oncogenic critical factors and is associated with a poor prognosis in certain types of cancer. For instance, it has been reported that elevated STAT3 expression is associated with poor prognosis in gastric cancer, lung cancer and live cancer [13]. Additionally, STAT3 has been revealed to be crucial involved in cancer cell proliferation, invasion, migration and apoptosis [14, 15].

A limited number of studies have previously demonstrated the clinical features of STAT3/ p-STAT3 in ESCC. Therefore, the present study investigated the association between STAT3 expression, p-STAT3 level and the clinical features of ESCC patients. Furthermore, the present study also investigated the potential prognostic value of STAT3/ p-STAT3 expression for predicting the survival of ESCC patients.

\section{Material and Methods}

\section{Patients and samples}

We obtained ESCC specimens from the 71 patients who were enrolled in this study at the department of thoracic surgery East Ward, Provincial Hospital Affiliated to Shandong University from September 2008 to January 2009. The inclusion criteria were as follows: 1) patients underwent complete resection and postsurgical pathology proved ESCC; 2 ) patients were diagnosed as postoperative pathologic stage I-III; 3) patients accepted no preoperative radiotherapy or chemotherapy; 4) patients had no serious surgical contraindication. The clinicopathalogical characteristics of the patients are listed in Table 1. This study was approved by Shandong University Ethics Committee. Adjacent non-tumorous esophageal tissues were used as the control tissues. Each specimen was divided into two parts. At least $0.5 \mathrm{~cm} \times 0.5 \mathrm{~cm} \times 0.5 \mathrm{~cm}$ ESCC specimens were used for western blot assay. The other ESCC specimen was used for histopathologic examination and immunohistochemistry.
Table 1. Correlation between STAT3/P-STAT3 expression and clinical characteristics of the ESCC (immunohistochemistry).

\begin{tabular}{|c|c|c|c|c|c|c|c|}
\hline \multirow{2}{*}{$\begin{array}{l}\text { Clinical } \\
\text { characteristics }\end{array}$} & \multirow[t]{2}{*}{ Patients } & \multicolumn{2}{|c|}{ STAT3 } & \multirow[t]{2}{*}{$P$} & \multicolumn{2}{|c|}{ p-STAT3 } & \multirow[t]{2}{*}{$P$} \\
\hline & & $\overline{(-)}$ & $(+)$ & & $(-)$ & $(+)$ & \\
\hline Gender & & & & ${ }^{*} 0.356$ & & & ${ }^{*} 0.489$ \\
\hline Male & 61 & 9 & 52 & & 22 & 39 & \\
\hline Female & 10 & 3 & 7 & & 5 & 5 & \\
\hline Age, years & & & & ${ }^{*} 1.000$ & & & 1.000 \\
\hline$\geq 60$ & 38 & 6 & 32 & & 14 & 23 & \\
\hline$<60$ & 33 & 6 & 27 & & 13 & 21 & \\
\hline Tumor length & & & & ${ }^{*}>0.05$ & & & ${ }^{*}>0.05$ \\
\hline$<3 \mathrm{~cm}$ & 7 & 2 & 5 & & 4 & 3 & \\
\hline $3-5 \mathrm{~cm}$ & 48 & 6 & 42 & & 16 & 32 & \\
\hline$>5 \mathrm{~cm}$ & 16 & 4 & 12 & & 7 & 9 & \\
\hline $\begin{array}{l}\text { Tumor } \\
\text { location }\end{array}$ & & & & ${ }^{*} 1.000$ & & & ${ }^{*} 0.461$ \\
\hline Middle & 42 & 7 & 36 & & 18 & 25 & \\
\hline Lower & 29 & 5 & 23 & & 9 & 19 & \\
\hline Differentiation & & & & ${ }^{*}>0.05$ & & & ${ }^{*}>0.05$ \\
\hline Well & 12 & 4 & 8 & & 5 & 7 & \\
\hline Moderately & 45 & 7 & 38 & & 17 & 28 & \\
\hline Poorly & 14 & 1 & 13 & & 5 & 9 & \\
\hline pT & & & & ${ }^{*}<0.01$ & & & ${ }^{*}<0.05$ \\
\hline pT1 & 4 & 3 & 1 & & 3 & 1 & \\
\hline pT2 & 46 & 7 & 39 & & 22 & 24 & \\
\hline pT3 & 21 & 2 & 19 & & 2 & 19 & \\
\hline $\mathrm{pN}$ & & & & ${ }^{*} 1.000$ & & & ${ }^{*} 0.005$ \\
\hline - & 45 & 8 & 37 & & 23 & 22 & \\
\hline+ & 26 & 4 & 22 & & 4 & 22 & \\
\hline pTNM & & & & ${ }^{*}<0.01$ & & & ${ }^{*}<0.05$ \\
\hline pI & 4 & 3 & 1 & & 3 & 1 & \\
\hline pII & 55 & 7 & 48 & & 23 & 22 & \\
\hline pIII & 12 & 2 & 10 & & 1 & 11 & \\
\hline
\end{tabular}

$P: \chi^{2}$ test; ${ }^{*}$ Fisher's exact probability test.

\section{Cell culture}

The human ESCC cell lines Eca-109 and Kyse-30 were obtained from the Cell Bank of Chinese Academy of Sciences (Shanghai, China). Cells were cultured in Dulbecco's Modified Eagle's Medium (DMEM; HyClone, Thermo Fisher Scientific, Waltham, MA, USA) contained $10 \%$ fetal bovine serum (FBS; Gibco, Thermo Fisher Scientific) and antibiotics (100U/mL, Sigma-Aldrich, Germany) at $37^{\circ} \mathrm{C}$ with $5 \% \mathrm{CO}_{2}$.

\section{Immunohistochemistry}

Immunohistochemistry staining for STAT3, p-STAT3, VEGF, CyclinD1, and Bcl-xl protein were detected by streptavidin-peroxidase method (SP method). Being fixed in 10\% neutral buffered formalin, the tissue specimens were processed routinely. Immunohistochemistry analysis was performed using rabbit antibody against human STAT3 (Spring Bioscience, USA), rabbit antibody against human p-STAT3 (tyr705) (Santa Cruz Biotechnology, USA), rabbit antibody against human VEGF (Zhongshanjinqiao Biotechnology, China), rabbit antibody against human Cyclin D1 (Zhongshanjinqiao Biotechnology), rabbit antibody against human Bcl-xL (Bioss Biotechnology, China), and visualized by the Envision System (Dako). STAT3, pSTAT3, VEGF, CyclinD1 and Bcl-xL were scored with 
immunohistochemistry using system as previously described [16, 17]. Briefly, a score of 3 indicated that $>50 \%$ of the cells exhibited mild to moderate staining intensity, or $>20 \%$ of cells exhibited strong staining intensity; a score of 2 indicated that $20-50 \%$ of cells demonstrated mild to moderate staining intensity, or $20 \%$ of cells exhibited strong staining intensity; a score of 1 indicated that $<20 \%$ of cells demonstrated mild to moderate staining intensity; and a score of 0 indicated that no staining was present. A score of $\geq 2$ demonstrated positive expression.

\section{Western Blot}

The micrograms of the proteins from each sample or Eca-109 and Kyse-30 cells treated with Stattic for $24 \mathrm{~h}$ were separated by SDS-PAGE and transferred to PVDF membranes (Millipore, France). The membranes were blocked with $5 \%$ skim milk and incubated with primary antibodies against STAT3 (Spring Bioscience, USA), p-STAT3 (S727; Santa Cruz, USA), VEGF (Proteintech Group, IL, USA), CyclinD1 (Proteintech Group), Bcl-xL (Proteintech Group), and $\beta$-actin (Bioss Biotechnology) at $4{ }^{\circ} \mathrm{C}$ overnight, following by incubation with HRP-conjugated secondary antibodies (Santa Cruz, USA) for $1 \mathrm{~h}$ at room temperature. Immunoblotted proteins were visualized by ECL reagents, and the signals were detected by Alphamager 2200 imaging system (Alphamager, USA) and Image J analysis software.

\section{Follow-up}

40 patients received postoperative chemotherapy, and 24 patients received postoperative radiotherapy. We examined the patients 3 to 4 months during the first 3 years and half a year thereafter. The patients only died of cancer were enrolled in the prognostic analysis.

\section{CCK8 assay}

For dose-dependent assay, cells were seeded into 96-well plates and treated with different concentrations of Stattic (0, 0.05, 0.1, 0.2, 0.3, 0.4, 0.5, $0.6,0.8,0.9$, and $1 \mu \mathrm{M}$, Med Chem Express, USA) for 24h. After treatment, $10 \mu \mathrm{l}$ of Cell Counting Kit-8 reagent (CCK8; Beijing Solarbio Science \& Technology, Beijing, China) were added into each well, following by incubation at $37^{\circ} \mathrm{C}$ for $90 \mathrm{~min}$. The optical density (OD) was measured at $450 \mathrm{~nm}$. Following treatment with Stattic of $24 \mathrm{~h}$, cell viability was measured at $0,24,48$, and $72 \mathrm{~h}$, respectively.

\section{Real-time polymerase chain reaction (RT-PCR)}

After being treated for $24 \mathrm{~h}$, total RNA from
Eca-109 and Kyse-30 cells was extracted using Ultrapure RNA Kit (CWBIO, Beijing, China) and reverse transcribed in complementary cDNA using the HiFiScript cDNA Synthesis Kit (CWBIO) according to the manufacturer's instructions. RT products were then used as templates to examine the expression of target gene mRNA using real-time PCR with the SYBR Premix Ex Taq II kit (Takara, Shiga, Japan). The primer sequences were as follows: VEGF, 5'- GCTACCTCAGCAAGACGTTATT -3' (forward), 5'- ATCGGCAGGAAGTGTGATTG -3' (reverse); CyclinD1, 5'-GTGCCACAGATGTGAAGT -3' (forward), 5'- GTAGGACAGGAAGTTGTTGG-3' (reverse); Bcl-xL， 5'- GGTGGTTGACTTTCTCTCC TAC -3' (forward), 5'- TCTCCGATTCAGTCCCTTCT -3' (reverse); $\beta$-actin, $5^{\prime}$ - CCCGAGCCGTGTTTCCT -3' (forward), 5'- GTCCCAGTTGGTGACGATGC -3' (reverse). $\beta$-actin was used as internal gene. The obtain data was analyzed according to the sample threshold cycle $(\mathrm{Ct})$ value from three independent experiments.

Table 2. Correlation between STAT3/P-STAT3 expression and clinical characteristics of the ESCC (Western blot).

\begin{tabular}{|c|c|c|c|c|c|c|}
\hline \multirow{2}{*}{$\begin{array}{l}\text { Clinical } \\
\text { characteristics }\end{array}$} & \multicolumn{3}{|l|}{ STAT3 } & \multicolumn{3}{|l|}{ p-STAT3 } \\
\hline & Protein Assay & $\mathrm{t}, \mathrm{t}^{\prime}$ & $P$ & Protein Assay & $\mathrm{t}, \mathrm{t}^{\prime}$ & $P$ \\
\hline Gender & & 0.607 & 0.546 & & 1.074 & 0.287 \\
\hline Male & $0.5426 \pm 0.2997$ & & & $0.4697 \pm 0.3270$ & & \\
\hline Female & $0.4800 \pm 0.3190$ & & & $0.3500 \pm 0.3240$ & & \\
\hline Age, years & & 0.352 & 0.726 & & 0.358 & 0.721 \\
\hline$\geq 60$ & $0.5459 \pm 0.3033$ & & & $0.4662 \pm 0.3476$ & & \\
\hline$<60$ & $0.5206 \pm 0.3024$ & & & $0.4382 \pm 0.3705$ & & \\
\hline Tumor length & & 0.958 & 0.389 & & 0.178 & 0.675 \\
\hline$<3 \mathrm{~cm}$ & $0.3857 \pm$ & & & $0.3286 \pm 0.3450$ & & \\
\hline $3-5 \mathrm{~cm}$ & 0.2854 & & & $0.4802 \pm 0.3137$ & & \\
\hline$>5 \mathrm{~cm}$ & $\begin{array}{l}0.5458 \pm 0.3039 \\
0.5625 \pm 0.2986\end{array}$ & & & $0.4250 \pm 0.3642$ & & \\
\hline $\begin{array}{l}\text { Tumor } \\
\text { location }\end{array}$ & & -0.848 & 0.399 & & -0.422 & 0.675 \\
\hline Middle & $0.5093 \pm 0.3146$ & & & $0.4395 \pm 0.3303$ & & \\
\hline Lower & $0.5714 \pm 0.2800$ & & & $0.4732 \pm 0.3267$ & & \\
\hline Differentiation & & 1.273 & 0.287 & & 0.653 & 0.524 \\
\hline Well & $\begin{array}{l}0.4417 \pm \\
0.3825\end{array}$ & & & $0.3583 \pm 0.3895$ & & \\
\hline Moderately & $\begin{array}{l}0.5289 \pm \\
0.2677\end{array}$ & & & $0.4800 \pm 0.2989$ & & \\
\hline Poorly & $\begin{array}{l}0.6286 \pm \\
0.3221\end{array}$ & & & $0.4464 \pm 0.3661$ & & \\
\hline pT & & 17.683 & 0.000 & & 7.764 & 0.001 \\
\hline pT1 & $0.1000 \pm 0.2000$ & & & $0.0750 \pm 0.1500$ & & \\
\hline pT2 & $0.4630 \pm 0.2322$ & & & $0.4022 \pm 0.3213$ & & \\
\hline pT3 & $0.7714 \pm 0.2849$ & & & $0.6357 \pm 0.2860$ & & \\
\hline $\mathrm{pN}$ & & -1.937 & 0.057 & & -3.757 & 0.000 \\
\hline- & $0.4822 \pm 0.2682$ & & & $0.3511 \pm 0.3335$ & & \\
\hline+ & $0.6231 \pm 0.3374$ & & & $0.6288 \pm 0.2300$ & & \\
\hline pTNM & & 17.761 & 0.000 & & 5.686 & 0.005 \\
\hline $\mathrm{pI}$ & $0.1000 \pm 0.2000$ & & & $0.075 \pm 0.1500$ & & \\
\hline pII & $0.4927 \pm 0.2387$ & & & $0.4364 \pm 0.3123$ & & \\
\hline pIII & $0.8667 \pm 0.2964$ & & & $0.6542 \pm 0.3130$ & & \\
\hline
\end{tabular}


A

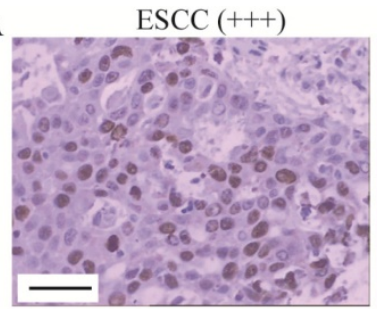

B

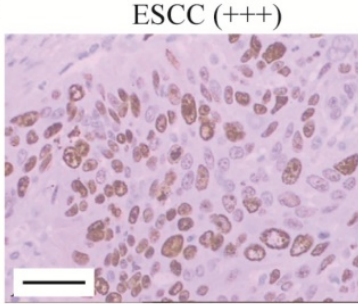

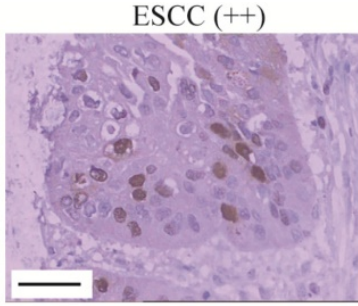

$\operatorname{ESCC}(+)$
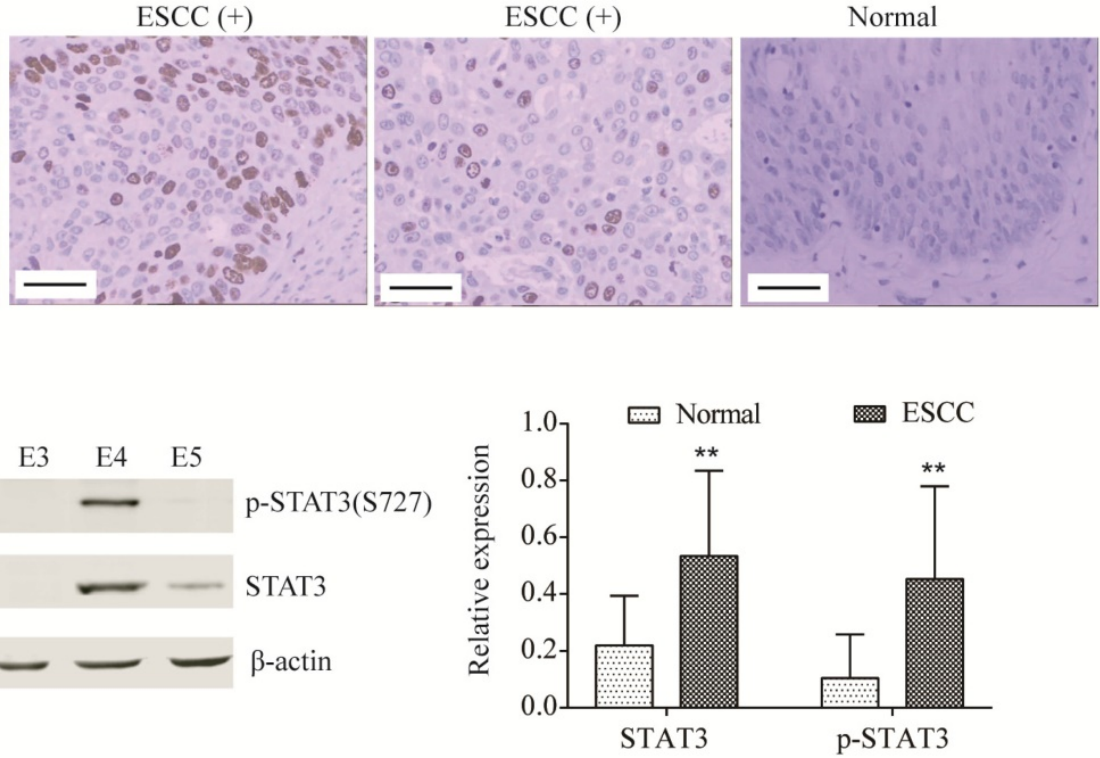

Figure 1. STAT3 and p-STAT3 was up-regulated in ESCC tissues. (A, B) STAT3 (A) and p-STAT3 (B) expression was determined by immunohistochemistry (Magnification 200). From left to right, ESCC tissues with strongly positive expression (+++), positive expression (++), weak positive expression (+) and normal tissue (control). Scale Bar=100 $\mu \mathrm{m}$. (C) STAT3 and p-STAT3 expression was determined by Western blot. N, normal tissue; E1, pT3 ESCC tissue; E2, pT2 ESCC tissue; E3, PT1 ESCC tissue; E4, ESCC tissue with lymphatic metastasis; E5, ESCC tissue without lymphatic metastasis. ** $P<0.01$ compared to normal.
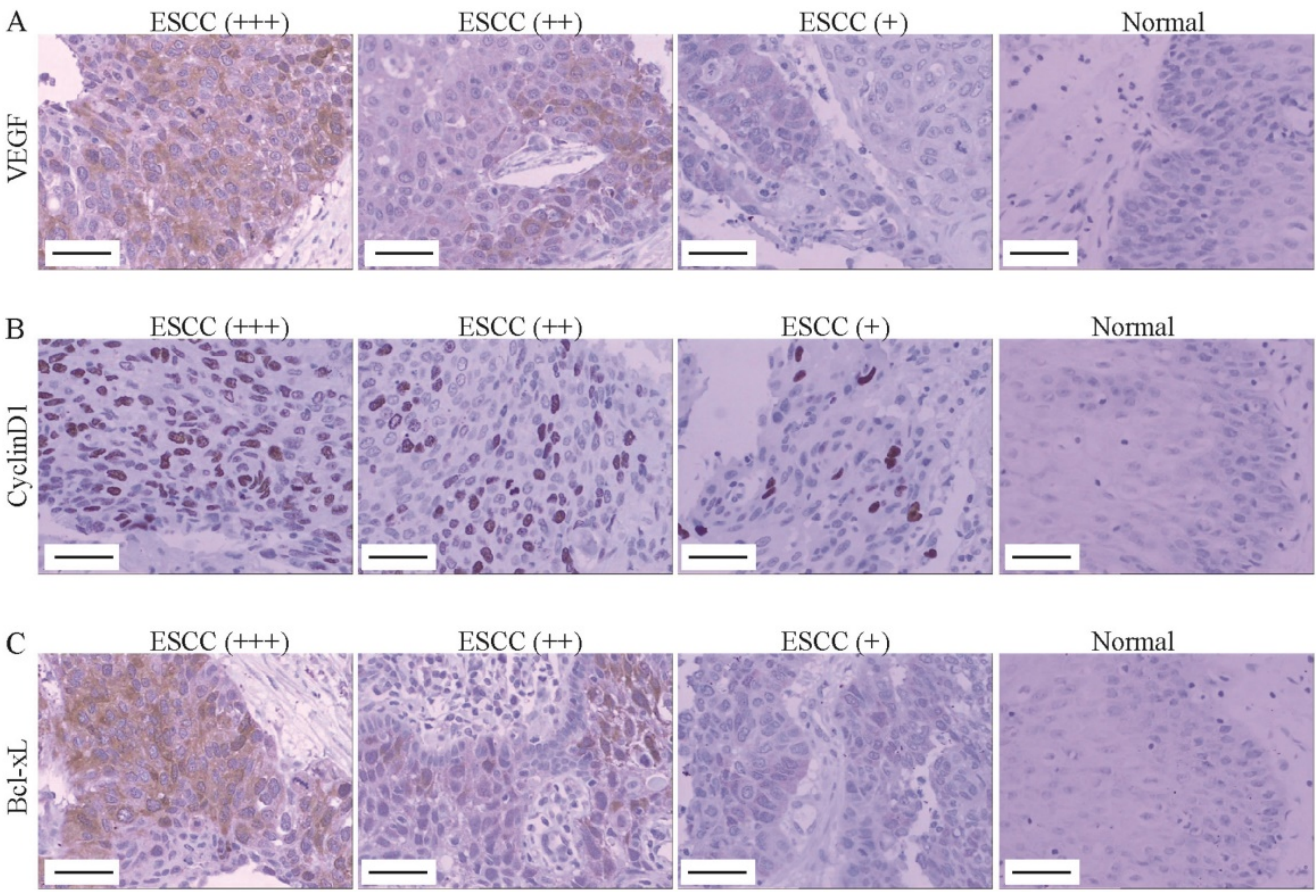

Figure 2. VEGF, Cyclin D1 and Bcl-xL was up-regulated in ESCC tissues. (A-C) The VEGF (A), Cyclin D1 (B) and Bcl-xl (C) expression was determined by immunohistochemistry (Magnification 200). From left to right, ESCC tissues with strongly positive expression (+++), positive expression (++), weak positive expression (+) and normal tissue (control). Scale Bar $=100 \mu \mathrm{m}$. 


\section{Statistical analysis}

Enumeration data were analyzed using $\chi^{2}$ test or Fisher's exact probability test. Measurement data was represented with Mean \pm standard deviation (SD) and t-rest. Univariate analysis was performed by modeling Kaplan-Meier survival curves. The log-rank test was used to calculate the survival rate. Multivariate analysis was carried out by the use of the Cox proportional hazard model. The difference between two groups were estimated with student's $t$ test. All statistical data were analyzed using SPSS (version 13; SPSS, Inc., Chicago, IL, USA). P<0.05 was considered to indicate a statistically significant difference.

\section{Results}

\section{Correlation between STAT3/ p-STAT3 expression and clinical features of ESCC}

As indicated in Figure 1A, the positive signal of STAT3 protein was located in cytoplasm and nucleus. The expression of STAT3 was correlated with infiltration degree (pT; pT1 $25.0 \%$ vs. pT2 $84.8 \%$ vs. pT3 90.5\%; $P<0.01$, Table 1) and pTNM stage (pI, $25.0 \%$ vs. pII, $87.3 \%$ vs. pIII, $83.3 \% ; P<0.01$, Table 1 ). The positive signal of $\mathrm{p}$-STAT3 protein, the active form of STAT3, was located in cell nucleus (Figure 1B). The level of p-STAT3 was correlated with pT (pT1 $25.0 \%$ vs. pT2 $52.2 \%$ vs. pT3 $90.5 \%$; $P<0.05$, Table 1 ), lymphatic metastasis ( $\mathrm{pN}$; $\mathrm{pN}-48.9 \%$ vs. $\mathrm{pN}+84.6 \%$; $P<0.01$, Table 1) and pTNM stage (pI, $25.0 \%$ vs. pII, $40.0 \%$ vs. pIII, $91.7 \% ; P<0.01$, Table 1 ). There was a positive correlation between STAT3 expression and $\mathrm{p}$-STAT3 in the cancerous tissue group $(\mathrm{r}=0.421$, $P<0.01$, Table 4).

The expression of STAT3 and level of p-STAT3 in ESCC tissues were also detected using Western blot (Figure 1C). Moreover, the expression of STAT3 was correlated with pT and pTNM stage (both $P<0.01$, Table 2), and the level of p-STAT3 was correlated with pT, pN and pTNM stage (both $P<0.01$, Table 2), which was consistent with immunohistochemical results.

Table 4. Correlation among STAT3/p-STAT3, VEGF, Cyclin DI and $\mathrm{Bcl}-\mathrm{xl}$ expression in ESCC (by immunohistochemistry).

\begin{tabular}{lll}
\hline & $\mathrm{r}_{\mathrm{s}}$ & $P$ \\
\hline STAT3 vs. p-STAT3 & 0.421 & 0.000 \\
STAT3 vs. VEGF & 0.255 & 0.032 \\
STAT3 vs. Cyclin D1 & 0.206 & 0.085 \\
STAT3 vs. Bcl-xl & 0.140 & 0.245 \\
p-STAT3 vs. VEGF & 0.352 & 0.003 \\
p-STAT3 vs. Cyclin D1 & 0.305 & 0.010 \\
p-STAT3 vs. Bcl-xl & 0.151 & 0.207 \\
VEGF $v$ s. Cyclin D1 & 0.193 & 0.107 \\
VEGF $v$ s. Bcl-xl & 0.047 & 0.695 \\
Cyclin D1 vs. Bcl-xl & 0.054 & 0.652 \\
\hline
\end{tabular}

Table 3. Correlation between VEGF, cyclinDI, Bcl-xL expression and clinical characteristics of the ESCC patients (Immunohistochemistry).

\begin{tabular}{|c|c|c|c|c|c|c|c|c|c|}
\hline \multirow[t]{2}{*}{ Clinical characteristics } & \multicolumn{2}{|c|}{ VEGF } & \multirow[t]{2}{*}{$P$} & \multicolumn{2}{|c|}{$\begin{array}{l}\text { CyclinD } \\
1\end{array}$} & \multirow[t]{2}{*}{$P$} & \multicolumn{2}{|c|}{ Bcl-xL } & \multirow[t]{2}{*}{$P$} \\
\hline & $(-)$ & $(+)$ & & $(-)$ & $(+)$ & & $(-)$ & $(+)$ & \\
\hline Gender & & & ${ }^{*} 0.298$ & & & ${ }^{*} 1.000$ & & & 0.733 \\
\hline Male & 23 & 38 & & 27 & 34 & & 21 & 40 & \\
\hline Female & 6 & 4 & & 4 & 6 & & 4 & 6 & \\
\hline Age, years & & & 1.000 & & & 1.000 & & & 0.146 \\
\hline$\geq 60$ & 15 & 22 & & 14 & 23 & & 13 & 24 & \\
\hline$<60$ & 14 & 20 & & 17 & 17 & & 12 & 22 & \\
\hline Tumor length & & & ${ }^{*}>0.05$ & & & ${ }^{*}>0.05$ & & & ${ }^{*}>0.05$ \\
\hline$<3 \mathrm{~cm}$ & 2 & 5 & & 1 & 6 & & 3 & 4 & \\
\hline $3-5 \mathrm{~cm}$ & 21 & 27 & & 22 & 26 & & 15 & 33 & \\
\hline$>5 \mathrm{~cm}$ & 6 & 10 & & 8 & 8 & & 7 & 9 & \\
\hline Tumor location & & & 0.809 & & & 0.808 & & & 0.316 \\
\hline Middle & 17 & 26 & & 18 & 25 & & 13 & 30 & \\
\hline Lower & 12 & 16 & & 13 & 15 & & 12 & 26 & \\
\hline Differentiation & & & ${ }^{*}>0.05$ & & & ${ }^{*}>0.05$ & & & ${ }^{*}>0.05$ \\
\hline Well & 5 & 7 & & 3 & 9 & & 5 & 7 & \\
\hline Moderately & 18 & 27 & & 21 & 24 & & 16 & 29 & \\
\hline Poorly & 3 & 11 & & 7 & 7 & & 4 & 10 & \\
\hline $\mathrm{pT}$ & & & ${ }^{*}<0.05$ & & & ${ }^{*}>0.05$ & & & ${ }^{*}<0.05$ \\
\hline pT1 & 3 & 1 & & 3 & 1 & & 3 & 1 & \\
\hline pT2 & 20 & 26 & & 20 & 26 & & 19 & 27 & \\
\hline pT3 & 3 & 18 & & 8 & 13 & & 2 & 19 & \\
\hline $\mathrm{pN}$ & & & ${ }^{*} 0.001$ & & & ${ }^{*} 0.012$ & & & ${ }^{*} 0.613$ \\
\hline 0.613 & 23 & 22 & & 25 & 20 & & 17 & 28 & \\
\hline+ & 3 & 28 & & 6 & 20 & & 8 & 18 & \\
\hline pTNM & & & $*<0.05$ & & & ${ }^{*}>0.05$ & & & ${ }^{*}>0.05$ \\
\hline pI & 3 & 1 & & 3 & 1 & & 3 & 1 & \\
\hline pII & 22 & 23 & & 25 & 30 & & 19 & 36 & \\
\hline pIII & 1 & 11 & & 3 & 9 & & 3 & 9 & \\
\hline
\end{tabular}

$P, \times 2$ test, ${ }^{*}$ Fisher's exact probability test.

\section{Correlation between STAT3/p-STAT3 expression and downstream proteins in ESCC}

Furthermore, the expression of downstream proteins of STAT3 was also assessed using immunohistochemistry assay. As indicated in Figure 2A, the positive signal of VEGF protein was located in cytoplasm, which was significantly correlated with $\mathrm{pT}$ (pT1 $25.0 \%$ vs. pT2 $56.5 \%$ vs. pT3 $85.7 \%$; $P<0.05$, Table 3), lymphatic metastasis ( $\mathrm{pN}$; $\mathrm{pN}-48.9 \%$ vs. $\mathrm{pN}+$ $88.5 \% ; P<0.01$, Table 3 ) and pTNM stage (pI, $25.0 \%$ vs. pII, $51.1 \%$ vs. pIII, $91.7 \% ; P<0.05$, Table 3$)$. The positive signal of Cyclin Dl protein was located in cell nucleus and up-regulated in ESCC tissues, which was significantly correlated with lymphatic metastasis ( $\mathrm{pN}$; $\mathrm{pN}-44.4 \%$ vs. $\mathrm{pN}+76.9 \% ; P<0.05$, Figure 2B, Table 3). The positive signal of $\mathrm{Bcl}-\mathrm{xL}$ protein was located in cytoplasm, which was significantly correlated with pT (pT1 $25.0 \%$ vs. pT2 $58.7 \%$ vs. pT3 $90.5 \% ; P<0.05$, Figure $2 C$, Table 3). Importantly, we found that there was a positive correlation between STAT3 protein expression and VEGF protein expression $(\mathrm{r}=0.255, \quad P<0.0$, Table 4$)$, but the expression of STAT3 was not correlated with the expression of cyclinD1 and Bcl-xL (both $P>0.05$, Table 4). Moreover, we found that the level of p-STAT3 was positively correlated with the expression of VEGF protein $(\mathrm{r}=0.352, P<0.01$, Table 4) and CyclinD1 
protein $(\mathrm{r}=0.305, P<0.05$, Table 4$)$ in the cancerous tissue group, except for Bcl-xL ( $P>0.05$, Table 4$)$.

\section{Inhibition of STAT3 activation inhibited downstream proteins expression in ESCC cells in vitro}

In order to further investigate the role of STAT3 in ESCC, two ESCC cell lines Eca109 and Kyse30 were treated with different concentrations of Stattic $(0,0.5$, $1,2,4,8,10$, and $20 \mu \mathrm{M})$ to inhibit the activation of STAT3. As indicated by CCK8 assay, Stattic inhibited the viability of Eca109 (Figure 3A) and Kyse30 (Figure 3B) cells in a dose-dependent manner. And the IC50 of Stattic was $5.532 \mu \mathrm{M}$ for Eca109 cells and $8.785 \mu \mathrm{M}$ for Kyse30 cells. As a result, $3 \mu \mathrm{M}$ of Stattic was used for the subsequent experiments for the appropriate effect on Eca109 cells and $5 \mu \mathrm{M}$ of Stattic for Kyse30 cells,
DMSO was used as the negative control (NC). Moreover, as shown in Figure $3 \mathrm{C}$ and D, Stattic also inhibited the viability of Eca109 and Kyse30 cells in a time-dependent manner. Our data demonstrated that compared to the NC group, the mRNA expression of VEGF, Cyclin D1 and Bcl-xl was significantly down-regulated in Stattic-treated Eca109 cells (both $P<0.05$, Figure 3E). Similar results were also observed in Kyse30 cells (Figure 3F). In addition, Western blot results further suggested that Stattic could obviously reduce the level of p-STAT3 and inhibit the expression of VEGF, Cyclin D1 and Bcl-xl in Eca109 and Kyse30 cells at protein level (both $P<0.05$, Figure 3G). Overall, these data indicated that blocking the activation of STAT3 can inhibit the growth of ESCC through down-regulation of VEGF, Cyclin D1 and Bcl-xl.
A

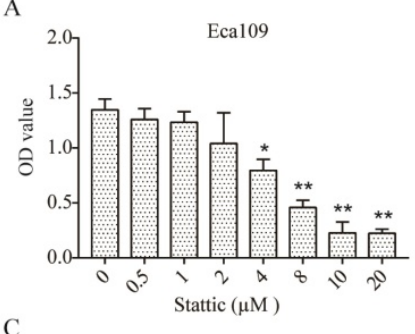

$\mathrm{C}$

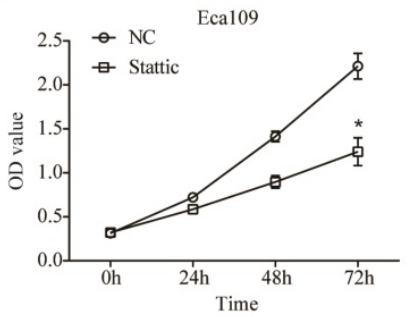

$\mathrm{E}$

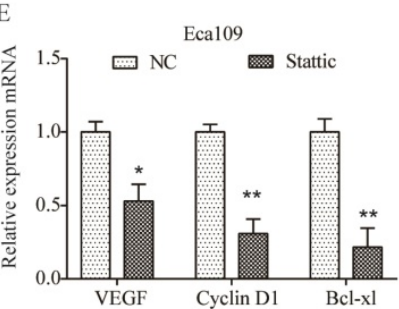

G

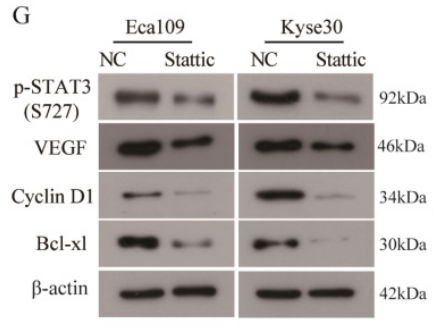

B
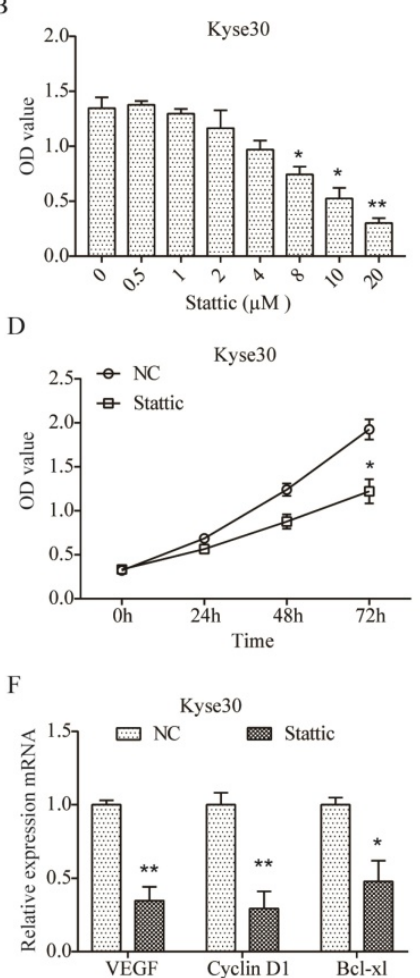
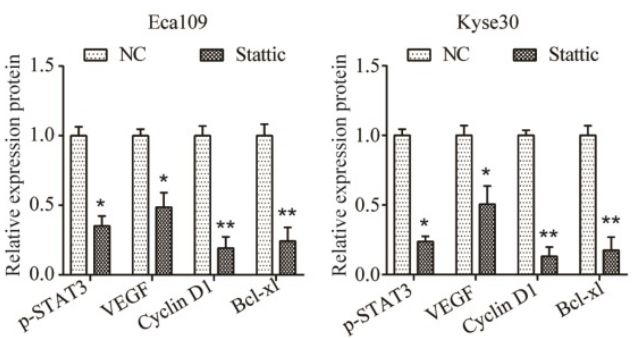

Figure 3. Stattic inhibited cell viability and expression of VEGF, Cyclin DI and Bcl-xl. (A, B) Eca 109 (A) and Kyse30 (B) cells were treated with different concentrations of Stattic for $24 \mathrm{~h}$, and CCK 8 assay was performed to assess cell viability. (C, D) Following treatment with Stattic for $0,24,48$ and 72 h, the viability of Eca109 (C) and Kyse30 (D) cells was examined using CCK8 assay. (E, F) After being treated with Stattic for 24 h, the expression of VEGF, Cyclin DI and Bcl-xL mRNA was detected using RT-PCR in Eca109 (E) and Kyse30 (F) cells. (G) The expression of p-STAT3, VEGF, Cyclin D1 and Bcl-xl protein was detected using Western blot in Eca 109 and Kyse 30 cells. NC, cells treated with DMSO; Stattic, cells treated with Stattic. All data from three independent experiments was quantified. ${ }^{*} P<0.05,{ }^{* *} P<0.01$ compared to NC. 

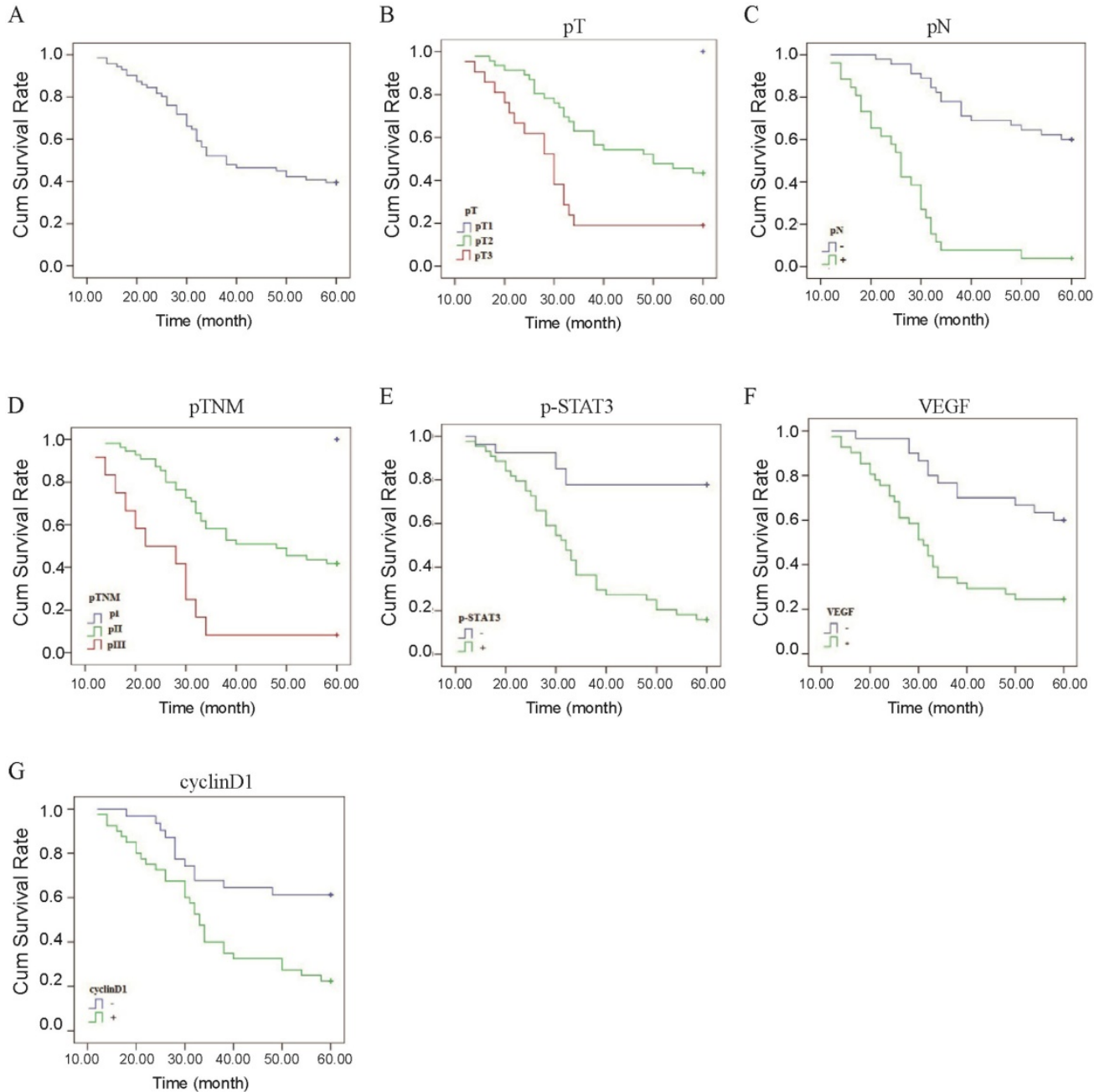

Figure 4. Correlation between p-STAT3/VEGF/cyclinD1 expression and prognosis of ESCC. (A) The Kaplan-Meier survival curve of 71 cases of patients with ESCC. (B) Survival curves of ESCC patients with different PT. Blue, patients with PT1; green, patients with PT2; red, patients with pT3. (C) Survival curves of ESCC patients with negative or positive $\mathrm{pN}$. Blue, patients with negative $\mathrm{pN}$; green, patients with positive $\mathrm{pN}$. (D) Survival curves of ESCC patients with different PTNM. Blue, patients with pl; green, patients with pll; red, patients with plll. (E) Survival curves of ESCC patients with positive and negative expression of p-STAT3 protein. Blue, p-STAT3 negative expression; green, p-STAT3 positive expression. (F) Survival curves of ESCC patients with positive and negative expression of VEGF protein. Blue, VEGF negative expression; green, VEGF positive expression. (G) Survival curves of ESCC patients with positive and negative expression of Cyclin DI protein. Blue, Cyclin DI negative expression; green, Cyclin DI positive expression.

\section{Correlation between STAT3/ p-STAT3 expression and prognosis of ESCC}

The Kaplan-Meier method indicated that the 5 -year survival rates of the 71 patients was $39.4 \%$ (Figure 4A). In univariate analysis by the log-rank test, the 5-year survival rate in patients after operation was significantly associated with $\mathrm{pT}(P<0.01$, Figure $4 \mathrm{~B}$, Table 5), pN $(P<0.01$, Figure $4 \mathrm{C}$, Table 5), pTNM stage $(P<0.01$, Figure $4 \mathrm{D}$, Table 5$)$, level of p-STAT3 $(P<0.01$, Figure $4 \mathrm{E}$, Table 5$)$, VEGF expression $(P<0.01$, Figure $4 \mathrm{~F}$, Table 5), and CyclinD1 expression $(P<0.01$, Figure 4G, Table 5). However, there were no statistically significant correlations with gender, age, tumor length; tumor location, histological differentiation, STAT3 expression, Bcl-xl expression, radiotherapy, and chemotherapy were demonstrated for the 5-year survival rate (Table 5). The results of
Cox regression multivariate analysis confirmed that $\mathrm{pN}$ and level of p-STAT3 were independent relevant factors (Table 6).

\section{Discussion}

STAT3 is regarded as a primary mediator of tumorigenesis and serves an important function in the proliferation, apoptosis and hyperplasia of tumor cell $[14,15]$. Constitutively activated STAT3 has been identified in certain types of cancer, including ESCC. In vitro, Wang et al. demonstrated that the STAT3 signaling pathway was constitutively activated in ESCC cells, and the expression of STAT3, VEGF and Bcl-2 were overexpressed in ESCC cell lines [18]. S Timme et al. reported that STAT3-regulated genes (STAT3, p-STAT3) were involved in ESCC cell proliferation and migration [19]. Activate STAT3 might affect STAT3 target genes expression and promote the 
growth of ESCC cells, which could be blocked by STAT3 inhibitor and specific siRNA $[20,21]$.

Table 5. Univariate analysis with respect to 5-year survival.

\begin{tabular}{|c|c|c|c|}
\hline Clinical characteristics & Patients (n=71) & 5-year survival (\%) & $P$ \\
\hline Gender & & & 0.169 \\
\hline Male & 61 & $36.1(22 / 61)$ & \\
\hline Female & 10 & $60.0(6 / 10)$ & \\
\hline Age (years) & & & 0.893 \\
\hline$\geq 60$ & 38 & $39.5(15 / 38)$ & \\
\hline$<60$ & 33 & $39.4(13 / 33)$ & \\
\hline Tumor length & & & 0.160 \\
\hline$<3 \mathrm{~cm}$ & 7 & $71.4(5 / 7)$ & \\
\hline $3-5 \mathrm{~cm}$ & 48 & $37.5(18 / 48)$ & \\
\hline$>5 \mathrm{~cm}$ & 16 & $31.3(5 / 16)$ & \\
\hline Tumor location & & & 0.886 \\
\hline Middle & 43 & $37.2(16 / 43)$ & \\
\hline Lower & 28 & $42.9(12 / 28)$ & \\
\hline Differentiation & & & 0.886 \\
\hline Well & 12 & $41.7(5 / 12)$ & \\
\hline Moderately & 45 & $37.8(17 / 45)$ & \\
\hline Poorly & 14 & $42.9(6 / 14)$ & \\
\hline pT & & & 0.001 \\
\hline pT1 & 4 & $100(4 / 4)$ & \\
\hline pT2 & 46 & $43.5(20 / 46)$ & \\
\hline pT3 & 21 & $19.0(4 / 21)$ & \\
\hline $\mathrm{pN}$ & & & 0.001 \\
\hline - & 45 & $60.0(27 / 45)$ & \\
\hline+ & 26 & $3.8(1 / 26)$ & \\
\hline pTNM & & & 0.001 \\
\hline $\mathrm{pI}$ & 4 & $100(4 / 4)$ & \\
\hline pII & 55 & $41.8(23 / 55)$ & \\
\hline pIII & 12 & $8.3(1 / 12)$ & \\
\hline STAT3 & & & 0.074 \\
\hline - & 12 & $66.7(8 / 12)$ & \\
\hline+ & 59 & $33.9(20 / 59)$ & \\
\hline p-STAT3 & & & 0.001 \\
\hline - & 27 & $77.8(21 / 27)$ & \\
\hline+ & 44 & $15.9(7 / 44)$ & \\
\hline VEGF & & & 0.001 \\
\hline - & 30 & $60.0(18 / 30)$ & \\
\hline+ & 41 & $24.2(10 / 41)$ & \\
\hline CyclinD1 & & & 0.002 \\
\hline - & 31 & $61.3(19 / 31)$ & \\
\hline+ & 40 & $22.5(9 / 40)$ & \\
\hline Bcl-xL & & & 0.114 \\
\hline - & 26 & $50.0(13 / 26)$ & \\
\hline+ & 45 & $33.3(15 / 45)$ & \\
\hline Chemotherapy, & & & 0.115 \\
\hline - & 31 & $51.6(16 / 31)$ & \\
\hline+ & 40 & $30.0(12 / 40)$ & \\
\hline Radiotherapy & & & 0.269 \\
\hline - & 47 & $44.7(21 / 47)$ & \\
\hline+ & 24 & $29.2(7 / 24)$ & \\
\hline
\end{tabular}

However, few studies reported the clinical and prognostic significance of STAT3 and p-STAT3 in ESCC patients. In Li's study, STAT3 protein expression was correlated with pTNM stage in ESCC patients, and dual high expression of STAT3 and Cyclin D1 predict worse survival outcome [11]. You et al. reported that p-STAT3 expression was correlated with lymph node metastasis, pTNM stage and metastatic status [22]. Huang et al. identified that p-STAT3 expression was significantly associated with poor prognosis in advanced esophageal cancer patients [23]. In the present study, a total of 71 ESCC patients enrolled the study, the STAT3 and p-STAT3 expression were detected by immunohistochemistry and Western blot at protein level. STAT3 was located in cytoplasm and nucleus while the p-STAT3 was exclusively observed in nucleus of ESCC cells. Moreover, our data demonstrated that the expression of STAT3 and p-STAT3 was both up-regulated in ESCC tissues, and the STAT3 expression was correlated with $\mathrm{pT}$ and pTNM stage, the p-STAT3 level was correlated with $\mathrm{pT}, \mathrm{pN}$ and pTNM stage. Xuan X et al. also reported that STAT3 and p-STAT3 were up-regulated in ESCC tissues, which was consistent with our results [24]. The findings described above indicated that STAT3 activation might increase the metastasis of ESCC.

Table 6. Results of cox regression multivariate 5-year survival analysis.

\begin{tabular}{lllllll}
\hline & $\mathrm{B}$ & $\mathrm{SE}$ & Wald & $\mathrm{P}$ & $\mathrm{HR}$ & $95.0 \%$ CI for HR \\
\hline Gender & -0.316 & 0.625 & 0.255 & 0.613 & 0.729 & $0.214 \sim 2.481$ \\
Age & -0.364 & 0.417 & 0.765 & 0.382 & 0.695 & $0.307 \sim 1.572$ \\
Tumor length & 0.648 & 0.375 & 2.987 & 0.084 & 1.911 & $0.917 \sim 3.982$ \\
Tumor location & -0.117 & 0.363 & 0.103 & 0.748 & 0.890 & $0.436 \sim 1.814$ \\
Differentiation & -0.053 & 0.308 & 0.030 & 0.862 & 0.948 & $0.519 \sim 1.732$ \\
pT & 0.459 & 0.622 & 0.544 & 0.461 & 1.582 & $0.467 \sim 5.357$ \\
pN & 1.306 & 0.589 & 4.906 & 0.027 & 3.690 & $1.162 \sim 11.718$ \\
pTNM & 0.284 & 0.812 & 0.122 & 0.727 & 1.328 & $0.271 \sim 6.520$ \\
STAT3 & 0.750 & 0.691 & 1.179 & 0.278 & 2.117 & $0.547 \sim 8.196$ \\
p-STAT3 & 1.158 & 0.551 & 4.414 & 0.036 & 3.183 & $1.081 \sim 9.372$ \\
VEGF & 0.761 & 0.414 & 3.386 & 0.066 & 2.141 & $0.952 \sim 4.818$ \\
cyclinD1 & 0.477 & 0.513 & 0.862 & 0.353 & 1.611 & $0.589 \sim 4.405$ \\
Bcl-xL & 0.630 & 0.445 & 2.008 & 0.156 & 1.878 & $0.785 \sim 4.492$ \\
Chemotherapy & -0.650 & 0.386 & 2.835 & 0.092 & 0.522 & $0.245 \sim 1.113$ \\
Radiotherapy & 0.090 & 0.408 & 0.049 & 0.825 & 1.094 & $0.492 \sim 2.432$ \\
\end{tabular}

STAT3 has been revealed to regulate the expression of many target genes in tumorigenesis and progression, including VEGF, Cyclin D1 and Bcl-xL [10, 24-26]. VEGF has been well known to play a crucial pro-oncogenic role in angiogenesis and tumor progression [27]. $\mathrm{Xu} \mathrm{Q}$ et al. reported that STAT3 is a key regulator of VEGF, targeting STAT3 can decrease the expression of VEGF [24]. Cyclin D1, an essential regulator of G1/S phase of cell cycle involved in cell proliferation, is frequently up-regulated and amplified in tumors. It is revealed that STAT3 can regulate the expression of Cyclin D1 through directly targeting its promoter in colorectal cancer cells [28]. Bcl-xL is a member of Bcl-2 family and functions as an anti-apoptotic protein. Bcl-xL is reported to be positively expressed in $46.8 \%$ of ESCC tissues [29]. In endometrioid adenocarcinomas, Wincewicz A et al. found that the expression of STAT3 is correlated with Bcl-xL [30]. In the current study, the expression of VEGF, Cyclin D1 and Bcl-xL was also assessed by immunohistochemistry. We observed that the expression of VEGF, Cyclin D1 and Bcl-xl was all up-regulated in ESCC tissues. Importantly, the level 
of p-STAT3 was all positively correlated to VEGF and Cyclin D1, except for Bcl-xL. Moreover, STAT3 expression was positively correlated to VEGF, but not with Cyclin D1 and Bcl-xL, which was not consistent with previous results in other cancers [28, 30]. Furthermore, blocking the activation of STAT3 by Stattic, a STAT3 inhibitor, could inhibit the viability of ESCC cells and down-regulate the expression of VEGF and Cyclin D1. Additionally, we observed that the expression of $\mathrm{Bcl}-\mathrm{xL}$ was also down-regulated by Stattic in Eca109 and Kyse30 cells, but its expression in tissues was not related to the expression of p-STAT3 (Table 4). The inconsistent results may be due to differences between tissues and cells, which will be further investigated in future study. Collectively, these results suggested that the activated STAT3 may promote the metastasis of ESCC by promoting the expression of VEGF and Cyclin D1.

It has been reported that STAT3 and p-STAT3 expression is associated with worse overall survival of many malignancies, including lung cancer, gastric cancer and hepatic cancer, but better prognosis of breast cancer [31]. Aleskandarany $\mathrm{M}$ A et al. also demonstrated that the nuclear p-STAT3 overexpression is positively associated with better prognosis of breast cancer [32]. In our study, to eliminate the impact of mixed factors on statistical analysis, we used both univariate and multivariate analysis to determine prognostic factors in order to make the results more objective. In this study, we observed that the 5-year survival rate of ESCC patients was $39.4 \%$, which was significantly associated with p-STAT3 expression, VEGF expression, Cyclin D1 expression, $\mathrm{pT}$, $\mathrm{pN}$, and pTNM stage. Additionally, $\mathrm{pN}$ and $\mathrm{p}-\mathrm{STAT} 3$ expression were relevant independent factors for a poor prognosis, which was consistent with the previous study that demonstrated that p-STAT3 expression was an independent prognostic factor for progressionfree-survival in ESCC [23]. Taken together, these findings indicated that activation of STAT3 could serve as a biomarker of poor prognosis in ESCC.

However, the present study had still several limitations. First, in China the indications for treatment not only depend on doctors' preferences but also on patients' willingness and economic status. These factors may have influenced the relatively poor survival result observed. In the study, 26 patients received postoperative chemotherapy, 10 patients received postoperative radiotherapy, and 14 patients received combined chemoradiotherapy. However, no statistically significant correlations with postoperative chemotherapy and radiotherapy were demonstrated for the 5-year survival rate both in univariate and multivariate analysis. Secondly, the study sample was relatively small. Lastly, all the patients' histologic type was squamous cell carcinoma in the study. Because ESCC is one of the most common malignant diseases in China, meaning that the patients enrolled in the study might not be representative of the population in the world.

In sum, STAT3 expression was correlated with pT and pTNM stage. The level of p-STAT3 was correlated with $\mathrm{pT}$, lymph node metastasis and pTNM stage. $\mathrm{pN}$ and p-STAT3 expression were relevant independent factors for the 5-year survival rate of ESCC patients. Activated STAT3 may promote the metastasis of ESCC by promoting the expression of VEGF and Cyclin D1. Collectively, these results suggest that p-STAT3 might serve as essential biomarker for tumor invasion and metastasis in ESCC.

\section{Abbreviations}

ESCC: Esophageal squamous cell carcinoma; STAT3: Signal transduction and activators of transcription factor 3; VEGF: Vascular endothelial growth factor.

\section{Acknowledgements}

The present study was funded by The Second Group of Jinan Science and Technology Development Program (grant no. 201602204) and Shandong Provincial Key Research and Development Program (grant no. 2017G006037).

\section{Competing Interests}

The authors have declared that no competing interest exists.

\section{References}

1. Sun ZG, Yu L, Yang F, Gao W, Wang Z, Zhu LM. Mucin 1 expression correlates with metastatic recurrence in postoperative patients with esophageal squamous cell cancer. Pol J Pathol. 2016; 67:384-391.

2. Hu D, Zhang M, Wang S, Wang Z. High expression of cyclooxygenase 2 is an indicator of prognosis for patients with esophageal squamous cell carcinoma after Ivor Lewis esophagectomy. Thoracic Cancer. 2016; 7: 310-315.

3. Wang D, Du L, Wang Z, et al. Association of fibroblast growth factor receptor 1 gene amplification with poor survival in patients with esophageal squamous cell carcinoma. Oncotarget.2017; 8:88857-88869.

4. Cheng JZ, Chen JJ, Xue K, Wang ZG, Yu D. Clinicopathologic and prognostic significance of VEGF, JAK2 and STAT3 in patients with nasopharyngeal carcinoma. Cancer Cell International.2018; 18:110.

5. Zhang L, Li J, Wang $\mathrm{Q}$, et al. The relationship between microRNAs and the STAT3-related signaling pathway in cancer. Tumour Biol.2017; 39:1010428317719869.

6. Han JJ, O'byrne M, Stenson MJ, et al. Prognostic and therapeutic significance of phosphorylated STAT3 and protein tyrosine phosphatase- 6 in peripheral-T cell lymphoma. Blood cancer journal. 2018; 8:110.

7. J.L. Geiger, J.R. Grandis, J.E. Bauman. The STAT3 pathway as a therapeutic target in head and neck cancer: Barriers and innovations. Oral Oncology. 2016; 56:84-92.

8. Santoni M, Conti A, Piva F, et al. Role of STAT3 pathway in genitourinary tumors. Future Science Oa. 2015; 1:1-9.

9. Lv J, Sun B, Mai Z, Jiang M, Du J. STAT3 potentiates the ability of airway smooth muscle cells to promote angiogenesis by regulating VEGF signaling. Experimental Physiology. 2017; 102:598-606.

10. Sulkowska U, Wincewicz A, Kanczuga-Koda L, Koda M, Sulkowski S. Comparison of E-cadherin with STAT3 and apoptosis regulators: Bak and 
Bcl-xL in endometrioid adenocarcinomas of different ER-alpha immunoprofile. Gynecol Endocrinol. 2018; 34:171-174.

11. Li H, Xiao W, Ma J, et al. Dual high expression of STAT3 and cyclinD1 is associated with poor prognosis after curative resection of esophageal squamous cell carcinoma. Int J Clin Exp Pathol. 2014; 7:7989-7998.

12. Johnson DE, O'Keefe RA, Grandis JR. Targeting the IL-6/JAK/STAT3 signaling axis in cancer. Nat Rev Clin Oncol. 2018; 15:234-248.

13. Igelmann S, Neubauer HA, Ferbeyre G. STAT3 and STAT5 activation in solid cancers. Cancers (Basel). 2019; 11(10): pii: E1428.

14. Zhou W, Bi X, Gao G, Sun L. miRNA-133b and miRNA-135a induce apoptosis via the JAK2/STAT3 signaling pathway in human renal carcinoma cells. Biomed Pharmacother. 2016; 8:722-729.

15. Yin Z, Zhang Y, Li Y, Lv T, Liu J, Wang X. Prognostic significance of STAT3 expression and its correlation with chemoresistance of non-small cell lung cancer cells. Acta Histochemica. 2012; 114:151-158.

16. Mizoguchi M, Betensky RA, Batchelor TT, Bernay DC, Louis DN, Nutt CL. Activation of STAT3, MAPK, and AKT in malignant astrocytic gliomas: correlation with EGFR status, tumor grade, and survival. J Neuropathol Exp Neurol. 2006;65:1181-1188.

17. Sun ZG, Zhang M, Yang F, Gao W, Wang Z, Zhu LM. Clinical and prognostic significance of signal transducer and activator of transcription 3 and mucin 1 in patients with non-small cell lung cancer following surgery. Oncology Letters. 2018; 15: 4278-4288.

18. Wang XH, Li SS, Yan AH, Lu CX, Guo YP. Constitutive activation of signal transducers and activators of transcription 3 and expression of its target gene products in human ESCC cell line. Nan Fang Yi Ke Da Xue Xue Bao. 2006;26:441-444

19. Timme S, Ihde S, Fichter CD2, et al. STAT3 expression, activity and functional consequences of STAT3 inhibition in esophageal squamous cell carcinomas and Barrett's adenocarcinomas. Oncogene.2014; 33:3256-3266.

20. Tian F, Yang X, Liu Y, et al. Constitutive activated STAT3 is an essential regulator and therapeutic target in esophageal squamous cell carcinoma. Oncotarget. 2017;8: 88719-88729.

21. Yang Y, Jin G, Liu H, et al. Metformin inhibits esophageal squamous cell carcinoma- induced angiogenesis by suppressing JAK/STAT3 signaling pathway. Oncotarget. 2017; 8:74673-74687.

22. You Z, Xu D, Ji J, Guo W, Zhu W, He J. JAK/STAT signal pathway activation promotes progression and survival of human oesophageal squamous cell carcinoma. Clinical \& Translational Oncology. 2012; 14:143-149.

23. Huang C, Wang L, Yang X, Lai L, Chen D, Duan C. Duan. Expression of activated signal transducer and activator of transcription-3 as a predictive and prognostic marker in advanced esophageal squamous cell carcinoma. World Journal of Surgical Oncology. 2015; 13:1-7.

24. Xuan X, Li S, Lou X, et al. Stat3 promotes invasion of esophageal squamous cell carcinoma through up-regulation of MMP2. Molecular Biology Reports. 2015; 42: 907-915.

25. Xu Q, Briggs J, Park S, et al. Targeting Stat3 blocks both HIF-1 and VEGF expression induced by multiple oncogenic growth signaling pathways. Oncogene. 2005; 24:5552-5560.

26. Luo J, Yan R, He X, He J. Constitutive activation of STAT3 and cyclin D1 overexpression contribute to proliferation, migration and invasion in gastric cancer cells. Am J Transl Res. 2017; 9:5671-5677.

27. Xie K, Wei D, Shi Q, Huang S. Constitutive and inducible expression and regulation of vascular endothelial growth factor. Cytokine Growth Factor Rev.2004; 15:297-324.

28. Qin A, Yu Q, Gao Y, et al. Inhibition of STAT3/cyclinD1 pathway promotes chemotherapeutic sensitivity of colorectal cancer. Biochemical \& Biophysical Research Communications.2015; 457:681-687.

29. Natsugoe S, Matsumoto M, Okumura H, et al. Bax and Bcl-X(L) expression are not related to prognosis in patients with advanced esophageal squamous cell carcinoma. Cancer Letters. 2001; 174: 91-97.

30. Andrzej W, Marek B, Luiza KK, et al. STAT3 and apoptosis regulators: Bak and Bcl-xL in endometrioid adenocarcinomas of different estrogen receptor- $\alpha$ immunoprofile. Gynecological Endocrinology.2011; 27:536-540.

31. Wu P, Wu D, Zhao L, et al. Prognostic role of STAT3 in solid tumors: a systematic review and meta-analysis. Oncotarget.2016;7 :19863- 19883.

32. Aleskandarany MA, Agarwal D, Negm OH et al. The prognostic significance of STAT3 in invasive breast cancer: analysis of protein and mRNA expressions in large cohorts. Breast Cancer Research \& Treatment.2016; 156:9-20. 